\title{
INDEFINITELY DESCENDING GROUND
}

\author{
Einar Duenger Bohn \\ University of Agder
}

[Forthcoming in Reality and its Structure, edited by R.Bliss \& G.Priest, OUP.]

We often say that some facts obtain in virtue of others, e.g. that semantic facts obtain in virtue of facts about language-use, or that normative facts obtain in virtue of descriptive facts, or that mental facts obtain in virtue of physical facts. The question I'm interested in is: must such in-virtue-of chains eventually end in some facts that don't obtain in virtue of any other facts? Or can they go on indefinitely without end $?^{1}$ In other words (to be clarified below), must the invirtue-of relation be well-founded?

In what follows, I argue that it must not, and point to some reasons for it even actually not being so. More specifically, in section 1, I introduce what is perhaps the closest we get to a standard notion of the in-virtue-of relation, namely a relation of grounding; in section 2, I argue that there is no good reason to think that this relation of grounding must be well-founded; and in section 3, I argue more directly that it's not necessarily well-founded, and further that there are reasons to think it's actually non-well-founded.

\section{The standard notion of grounding}

Everything about in-virtue-of-talk can and has been questioned, but the following is perhaps the closest we get to a standard underlying notion these days (Rosen, 2010; Bliss \& Trogdon 2014; Raven 2015). Saying that a fact obtains in virtue of some others is to say that the fact is grounded in those other facts. Such grounding is taken to be a one-many relation between one fact and a plurality of (one or more) facts, imposing what we might (with some slack) call a strict partial order on its domain: ${ }^{2}$ no fact even partially grounds itself (irreflexivity); if a fact $\mathrm{p}$ is grounded in some facts qq, then no one of qq is even partially grounded in $\mathrm{p}$ (asymmetric); and if a fact $\mathrm{p}$ is grounded in some facts $\mathrm{q}, \mathrm{rr}$, and $\mathrm{q}$ is grounded in some facts ss, then $\mathrm{p}$ is grounded in $\mathrm{rr}, \mathrm{ss}$ (transitivity). Also, if a fact $p$ is grounded in some facts qq, then, necessarily, if qq obtain, then $p$ obtain (necessitation); if a fact $\mathrm{p}$ is grounded in some facts $\mathrm{qq}$, then is not the case that for any $\mathrm{r}, \mathrm{p}$ is grounded in qq,r (non-monotonicity); and if a fact $\mathrm{p}$ is grounded in some facts $\mathrm{qq}$, then $\mathrm{qq}$ metaphysically explain $\mathrm{p}$ (explanatoriness). ${ }^{3}$ The latter kind of metaphysical explanation amounts to constitutively explaining what a fact consists in. The underlying notion of grounding is a notion of full as

\footnotetext{
${ }^{1}$ Note that there can be infinite chains that are limited, but I wish to talk about infinite chains that are unlimited; I here and throughout use the term 'indefinite' for that purpose. This should not be confused with the way 'indefinite' is sometimes used in the philosophy of mathematics, where there is a constructional or potential aspect to it, nor should it be confused with the way 'indefinite' is sometimes used in debates over vagueness, where there is an aspect of, well, vagueness to it.

2 I thus adopt the so-called predicate approach, not the operator approach. I also assume grounding to be factive.

3 One might here distinguish between, on the one hand, grounding being explanation, and on the other hand, grounding backing explanation. I assume the former, unless noted otherwise.
} 
opposed to partial grounding, where a fact $\mathrm{p}$ is partially grounded in some facts $\mathrm{qq}$ iff there are some facts rr such that $\mathrm{p}$ is fully grounded in $\mathrm{rr}$ and $\mathrm{qq}$ are among rr; $\mathrm{p}$ being fully grounded in $\mathrm{rr}$ being our official primitive, but intuitively characterized as providing a complete metaphysical explanation of $\mathrm{p}$.

I henceforth call this the standard notion of grounding (SNG). I will not further discuss or defend SNG or any of its abovementioned features. ${ }^{4}$ Instead, I will here simply assume SNG in order to argue against it being necessarily wellfounded, in favor of some reasons for it actually being non-well-founded.

What is the notion of well-foundedness in play? There has been some ambiguity in the literature with respect to what it is more exactly, but my arguments below will be directed at the following notion identified in Schaffer (2009, 2010), and made more precise in Dixon (forthcoming) and Rabin \& Rabern (forthcoming): ${ }^{5}$ every non-fundamental fact $p$ is fully grounded in some fundamental facts $q q$, where a fact $\mathrm{q}$ is fundamental iff there are no facts rr such that q is (partially or fully) grounded in rr. ${ }^{6}$ I henceforth call this notion of wellfounded grounding WF, regimented as follows:

$(\mathrm{WF}): \forall p(\sim F p \rightarrow \exists q q(F q q \wedge q q G p))$

where 'qq' range over pluralities of (one or more) facts, 'p' over single facts, ' $F$ ' expresses being fundamental, and ' $G$ ' expresses grounding. ${ }^{7}$ The question then is simply this: must SNG obey WF?

\section{Why believe grounding must be well-founded?}

What are the arguments for WF? There aren't many. It seems to be more of an assumed metaphysical axiom (or metaphysical law) supported by intuition. The underlying intuition is perhaps most forcefully identified and endorsed in Schaffer (2009:376, 2010:37), where he claims that ' $t$ t]here must be a ground of being. If one thing exists only in virtue of another, then there must be something from which the reality of the derivative entities ultimately derives.' Schaffer (2010:62) further claims that if there is no ultimate ground, then '[b]eing would be infinitely deferred, never achieved.' Finally, Schaffer (forthcoming: section 4.5) claims that '[g]rounding must be well-founded because a grounded entity inherits its reality from its ground, and where there is inheritance there must be a source.' And (ibid): 'the grounded exists in virtue of its ground. This is why a

\footnotetext{
${ }^{4}$ For references to further discussions on each of these features, see Bliss \& Trogdon (2014) and Raven (2015). For a general criticism of an overall notion of grounding, see Wilson (2014) and Koslicki (2015). See also Dasgupta (2014a), where grounding is argued to be many-many, rather than one-many.

5 Note that the following notion of well-foundedness is not the mathematical (settheoretical) notion of well-foundedness. The above well-foundedness of grounding is a distinctively metaphysical notion. Note also that this is just one among several notions of metaphysical well-foundedness. Though I think much of what I go on to say does not hinge on this particular notion, my conclusions should be hedged accordingly. ${ }^{6}$ See Dasgupta (2014b) for some problems with the above definition of fundamentality. Fortunately, those problems will not affect my overall argument in this paper. ${ }^{7}$ I take ' $F$ ' to be distributive, but see fn.13, section 2 below.
} 
source of reality is needed, in order for there to be anything to transfer.' ${ }^{8}$ Cameron (2008:6) too finds Schaffer's intuition appealing (though he ultimately rejects that it must hold in favor of it only most likely actually holding for methodological reasons): 'if we never reach a bottom level, then it is hard to see why there are any complex objects at all.'9

The intuition thus seems to be something like this: reality, or being is transferred from the ground to the grounded, so all facts gain their being from their ground, so if there is no bottom ground, there is nowhere from which the transfer of being initially comes, nowhere from which to gain being to begin with, so SNG must obey WF on pain of there not being any (being to) facts at all.10

Here is one way to make the intuition into an argument for WF: by definition, something is a fundamental fact if and only if it has no ground; hence, if there are no fundamental facts, all facts have ground; and if all facts have ground, there are no facts; but obviously there are some facts; hence there are some fundamental facts. Let's call this the argument for WF.

The argument is valid; the main question is why we should believe the next to last premise: if all facts have ground, there are no facts? I for one simply don't feel the intuitive pull here. ${ }^{11}$ An intuition pump might come by the more or less dynamical metaphors often used to explain the grounding relation: the ground transmits its being to the grounded, the grounded gains, achieves, or derives its being from the ground. So, if there is no bottom ground, there is nothing that can thus transmit being to the grounded, nothing from which the grounded can thus gain, achieve or derive its being. The dynamical talk makes this sound somewhat plausible. It's as if you are to fill up a swimming pool by the use of a hose: if nothing comes out of that hose, then of course the swimming pool will not be filled up. Or, at least in my experience, if I don't at some definite point start writing my paper, it just won't be written. But the problem is that grounding is nothing like such dynamical processes. There is no such definite dynamical starting point of the grounding chain. Grounding is like a synchronic, static mathematical relation (like in arithmetic), not like a diachronic, dynamic physical relation (like in thermodynamics, or action theory). Grounding is an explanation of what the obtaining of a fact consists in, atemporally; grounding is not an explanation of the causal history of that fact (cf. Fine, 2001, 2012; Rosen, 2010). So, any intuitive pull we might feel from the dynamical metaphors is of no help in a defense of SNG necessarily obeying WF.

Now, before I look at other ways to try to defend the above premise in the argument for WF, consider the argument that simply drops it: by definition, something is a fundamental fact iff it has no ground; hence, if there are no

\footnotetext{
8 Thomas Aquinas' (1266-8/1993:200-202), in his five ways towards the necessary existence of God, shares Schaffer's intuition concerning the need for a source of being. In general, there are great similarities between Aquinas' foundationalism in his five ways and Schaffer's foundationalism with respect to grounding.

${ }^{9}$ Note: Schaffer doesn't and Cameron might not endorse the full package of SNG, but officially I'm here only interested in whether SNG in particular must be well-founded (though I do believe my arguments generalize to other notions of grounding as well). 10 For more on this picture of transference, inheritance, or source of being, see Trogdon (ms). I treat the notions of reality and being as interchangeable, but try to keep the notion of existence separate; though in fact nothing hinges on this for present purposes. 11 And, in any case, why believe my intuitions match deep metaphysical truths?
} 
fundamental facts, all facts have ground. Now that is a solid conditional argument for what I henceforth call indefinitely descending ground:

\section{(IDG): $\forall p \exists q q(q q G p)$}

Given our assumption that $\mathrm{G}$ is a strict partial order as per SNG, IDG gives us infinite, non-ending chains of grounding. ${ }^{12}$ Given some very minor, plausible assumptions, IDG is incompatible with WF. Proof: assume WF and $\sim$ Fp. Then, $\exists \mathrm{qq}(\mathrm{Fqq} \wedge \mathrm{qqGp})$. Let $\mathrm{q}_{1}$ be one of those $\mathrm{qq}$ and assume the distributivity of $\mathrm{F}$. It then follows that $\mathrm{Fq}_{1}$; but by definition of fundamentality, $\forall \mathrm{p}(\sim \mathrm{Fp} \leftrightarrow \exists \operatorname{ss}(\mathrm{ssGp}))$; hence $\sim \exists s s\left(s_{s G q}\right)$; but by IDG, $\exists q q\left(q_{q G q}\right)$; hence, contradiction. Now, the minor assumptions that fundamentality is a distributive property and the definition of fundamentality is here taken for granted (though neither one is beyond dispute ${ }^{13}$ ), so WF and IDG are incompatible.

Now, one way to indirectly defend the controversial premise in the above argument for WF (i.e. the premise that if all facts have ground, there are no facts) is to argue that IDG amounts to an appropriately vicious infinite regress. I take it a benign infinite regress won't do (for example, it 's not a problem that we have: 's' is true; 's' is true' is true; '"s' is true' is true' is true; and so on ad infinitum). I also take it a mere infinite regress won't do (for example, it's not a problem that zero has infinitely many successors). Rather, IDG must amount to an infinite vicious regress, and it must be of the appropriate kind to support the argument for WF. But what could such an appropriately infinite vicious regress be in this case?

I can think of at least two candidates (adapted from Nolan, 2001; Bliss, 2013). The first candidate deals with reductive explanations. Now, 'reduction' is said in many ways, but assume SNG is reductive in the sense that if qqGp, then qq explain away $\mathrm{p}$ as being in some sense non-real. Given IDG, one would then explain away facts, but never explain them away in terms of something that is not thus further explained away. One might then get the feeling that every fact $p$ is somehow explained away, but into nothing, so to speak; that every fact is 'infinitely deferred, never achieved' (Schaffer, 2010:6314).

But, first, why think grounding is thus explanatorily reductive? It is not a common view of grounding (Fine, 2001, 2012; Schaffer, 2009, 2010; Rosen, 2010). In fact, much initial motivation for appealing to grounding is to be able to truly say that some things obtain without thereby admitting that they are as real as all other things obtaining (Schaffer, 2009). For example, we want to say - truly

12 Cf. Bliss (2013); Tahko (2014); Morganti (forthcoming). With G being transitive but failing irreflexivity, we could have loops of ground; see Bliss (2014). I'm not in principle opposed to such loops, but SNG is for present purposes assumed to be irreflexive, so, by assumption, no loops.

13 If there are fundamental facts, but fundamentality is a collective property, things will look slightly different. Though that is an interesting idea, I will ignore it in what follows since I believe that there most likely are no fundamental facts. See Dasgupta (2014b) for criticism of the notion of fundamentality.

14 Though note that to the extent one is concerned with grounding objects rather than facts, the notion of grounding seems to fail the assumption of explanatoriness, in which case one loses this particular defense of his intuition. One must then, as e.g. Schaffer (forthcoming) does, appeal to a notion of grounding that backs explanation, without grounding itself being explanation. 
- that there are tables and chairs, but not thereby admit that tables and chairs are as fundamental as particles. So, as far as I can tell, a proponent of IDG could and should simply reject that grounding is explanatorily reductive. Grounding is explanatory, but non-reductive. ${ }^{15}$ Grounding metaphysically explains a given fact in terms of other facts, but grounding does not thus explain away that fact as somehow non-real. ${ }^{16}$

Second, assume grounding is thus explanatorily reductive. Then the above argument, corresponding intuition and supposed vicious regress get things backwards. By SNG, the grounded has its being (or nature, or existence) in virtue of the ground. But if so, the ground surely cannot have less being than the grounded. Either the ground has the same degree of being as the grounded, or the ground has more being than the grounded. But then, as we approach infinity towards ground, we either stay with the same degree of being, or we approach infinite being! In neither case is being infinitely deferred, never achieved, as per Schaffer's intuition. If anything, being is always deferred, but infinitely achieved! So, if grounding is reductive, we're not explaining facts away only to approach nothingness, but rather we're explaining facts away in terms of other facts that have equal or more being. ${ }^{17}$

Now, one might object that infinite being makes no sense, so at best we have an equal degree of being along the grounding chain. But then, as we go down the grounding chain, being is infinitely deferred, never achieved because without WF that equal degree of being is zero! But, of course, that would be begging the question.

It is very important not to confuse various perspectives of the supposed explanatoriness involved in SNG (cf. Bliss, 2013; Morganti, forthcoming). Assume IDG, its corresponding chains of grounding, and the corresponding totality of all such chains of grounding, which I'll call the chains of being. ${ }^{18}$ We then have three questions, what we might call a local, a regional, and a global question. Local Question (LQ): for any fact p, what grounds p? Regional Question (RQ): what

\footnotetext{
${ }^{15}$ One might of course still accept Rosen's (2010:123) principle that if $p$ reduces to qq, then $\mathrm{qqGp}$, without accepting that grounding is reductive in the above sense.

${ }^{16}$ Note that this latter notion of being real is not necessarily Fine's (2001) notion of being real. Note also that to the extent that Fine's notion of being real is taken as a guide (though no guarantee) to being fundamental, Fine's (2001:26) definition of reduction is incompatible with IDG.

${ }^{17}$ See also Fine (2001:27 and fn.38). Morganti (forthcoming) argues that rather than disappearing as per the above intuition, being emerges from infinite grounding chains. Cameron (2008:10) too raises a similar point: 'Why could not everything get a bit more real as we progress down the chain, without anything being wholly real?' But his intuition 'rules this out', though, as he himself points out, 'this just is the intuition that there must be a fundamental level.' Note that an appeal to an analogy with causation doesn't seem to help here: there seems to be no more to an effect than what's in its cause(s). In fact, since WF postulates unexplained facts at the bottom, it seems to me it's ultimately WF that fails to explain the being of our facts, not IDG. See section 3 below. See also Tahko (2014).

18 Recall, grounding is supposed to impose a partial order, not a total order, so the chains of being need not be a single connected grounding chain, but can be many disconnected ones.
} 
grounds the chain of grounding of $\mathrm{p}$ ? $^{19}$ Global Question (GQ): what grounds the chains of being? A proponent of IDG should commit to answering LQ, and might or might not commit to answering RQ (cf. Dasgupta, 2014b). But should she commit to answering GQ? No! Given SNG+IDG, GQ is incoherent. First of all, the chains of being is presumably a plurality of facts, not a single fact, which is what it needs to be to be grounded as per SNG. Second, assume the chains of being amount to an all-encompassing global fact; call it g. Then, by IDG, there are some facts that ground g; but, by SNG being a strict partial order, those facts must be distinct from g; so $\mathrm{g}$ is not an all-encompassing global fact, contradicting the initial assumption. So, we might put it like this: each fact individually has a ground, but all facts taken together (speaking unrestrictedly!) cannot have a ground.

One might object that by invoking many-many grounding (as per Dasgupta, 2014a), one has the logical resources to answer GQ. But note first that one is then changing the assumptions of the argument, by switching from SNG to another slightly different notion of grounding, call it SNG*. Second, that might be the right thing to do, perhaps for independent reasons too, but, given IDG, it does not help answering GQ. Assume the chains of being amount to an allencompassing plural global fact; call it gg. Then, by IDG, there are some facts that ground gg; but, by SNG* being a strict partial order, those facts must be distinct from gg; so gg is not an all-encompassing plural global fact, contradicting the initial assumption. So, this time, we might put it like this: each fact individually has a ground, but all facts taken collectively (speaking unrestrictedly!) cannot have a ground.

Neither does it help to invoke Fine's (2012) distinction between being ungrounded and being zero-grounded. We might put the distinction like this: something is zero-grounded iff it is grounded, but in the empty collection of facts, and something is ungrounded iff it is not grounded at all. Now, SNG is assumed to be explanatory, so while being ungrounded amounts to having no explanation, being zero-grounded amounts to having an explanation, but in terms of the empty collection of facts. But, I say, a metaphysical explanation of a fact in terms of the empty collection of facts is no metaphysical explanation of that fact, at least not as per SNG. Saying that the obtaining of a fact p consists in the obtaining of the empty collection of facts, is not metaphysically explaining $p$ unless $p$ is the empty fact. So, by trying to answer GQ by invoking zero-ground, one either fails due to not being appropriately explanatory, or one must be invoking a different notion of grounding from SNG. ${ }^{20}$ So, assuming SNG, invoking Fine's (2012) distinction between being ungrounded and being zero-grounded just does not help, at least not on pain of changing the assumptions. Of course, maybe that is the right thing to do in the end, but not for present purposes. ${ }^{21}$

\footnotetext{
${ }^{19}$ Note that RQ is not the question of what grounds a grounding fact. The question of what grounds a grounding fact falls under $L Q$.

20 Presumably, one could do better with respect to zero-grounding by switching to an operator approach towards $\mathrm{G}$, rather than our predicate approach. But then, again, one is moving away from SNG, which is our present concern.

${ }^{21}$ See Litland (forthcoming) for some further work on zero-grounding. Unfortunately, Litland's assumptions are difference from ours (e.g. G is a non-factive operator), so his account does not obviously help answering GQ. Despite his insistence to the contrary, I
} 
Admittedly, the above argument is quick, but, whether or not it's too quick, there is another more decisive argument for not invoking zero-grounding to assist us with respect to GQ: by our present assumptions, it entails necessitism, the view that all actual facts are necessary. ${ }^{22}$ Assume the chains of being are zero-grounded, i.e. grounded in the empty collection of facts. Then, since the empty collection of facts necessarily exists, by the assumption of necessitation, all actual facts exist necessarily; hence necessitism. But, I claim, necessitism is false, so the chains of being are not zero-grounded.

So, I conclude, whether grounding is reductive or not, by IDG, each fact has a ground, and hence a metaphysical explanation in terms of some other facts, and no fact is thereby lost into nothingness; i.e. no fact is 'infinitely deferred, never achieved'. Given IDG (and SNG, or SNG*), there is no sensible question about what the ground of being as such is; the global question (GQ) of what grounds the chains of being themselves is incoherent.

The point that LQ (and maybe RQ) is what we want answered, with GQ being incoherent, resembles a point made by Hume (1779:IX) in his objection to cosmological arguments for the existence of God. According to Hume, if one has explained each step in a perhaps infinite causal chain, there is nothing more to be explained, and in particular no need to postulate a first cause to explain it all. Now, Pruss (2012:81-82) objects to Hume by the following example. Consider the flight of a cannonball between 12:00 and 12:01, and let $\mathrm{p}_{\mathrm{t}}$ be the state of the cannonball at time $t$. Let $p$ be the conjunction of all $p_{t}$ such that 12:00<t<12:01. Then each $p_{t}$ might explain its successor $p_{t+}$, such that each $p_{t}$ is explained, but still, Pruss claims, the flight of the cannonball itself is not explained, so Hume is wrong.

Is there a similar objection in the vicinity with respect to IDG? No. The flight case is misleading because there is an external perspective on the flight of the cannonball, but there isn't any such external perspective on all the chains of being (witnessed by GQ being incoherent). So, adapted to our global case of grounding, I think Hume is right.

The second candidate for a vicious regress in indirect support of the controversial premise in the argument for WF is similar to the prior one in terms of reductive explanations, but claims that there is another kind of failure. Whether grounding is reductive or not, the supposed failure can be seen by analogy with the toy example of the homunculus theory of perception (Cf. Nolan, 2001; Bliss, 2013). Suppose someone attempts to explain the perception of the fact that $\mathrm{p}$ as follows. There are outside signals coming into the eye, received by a homunculus sitting on the inside of the eye, who interprets the signals as being of the fact that $\mathrm{p}$ before sending them off to the brain. How does the homunculus so interpret the signals? Well, there is another homunculus sitting inside the first homunculus, who interprets the signals as being of the fact that $\mathrm{p}$ before sending them off to the first homunculus. How does the second homunculus so interpret the signals? Well, there is a third homunculus sitting inside the second homunculus... and so on ad infinitum. Obviously, here the supposed explanation

also fail to see how Litland's account of zero-grounding is metaphysically explanatory, but let that be as it may.

22 I owe this argument to Jon Litland, though of course he is not to blame. Note that the necessitism in play is not to be confused with our earlier assumption of necessitation. 
of the perception that $\mathrm{p}$ fails; we might plausibly blame the explanation for creating a vicious regress.

Now, the worry this time is not so much that we explain away facts into nothingness, but that the explanation fails in the sense of having to re-ask the same question with respect to the same kind of object over and over again ad infinitum. The explanation thus gets us nowhere, so to speak. But there is no such problem in our case of grounding. IDG does not create any such vicious regress. If qqGp, then, by SNG, qq provide a full metaphysical explanation of $p$, so there is no further question about what grounds p. So, we do get somewhere. We do of course have the further question of what grounds the facts among qq (not to mention what grounds the fact that $\mathrm{qqGp}$ ), and so on ad infinitum, but that is not analogous with the homunculus theory of perception. In the homunculus-case, one has the same content over and over again, but not so in the IDG-case of grounding. To make the homunculus-case more analogous to the IDG-case, we would have to explain the perception that $p$ in terms of the first homunculus, but then explain this homunculus in terms of something non-homunculus-like, or at least something different-hounculus-like and so on ad infinitum. But then the reason for the initial failure in explanation has gone away. The analogy simply doesn't hold up to scrutiny.

In general, we might say that an infinite regress is vicious if something we want to explain cannot be explained because of the regress (Nolan, 2001; Bliss, 2013), so, in the particular case of grounding, IDG's infinite regress is vicious if something we want to ground cannot be grounded because of it. But, as we have just seen, given IDG, it makes no sense to ask for the ground of the chains of being themselves, i.e. there is no "global" fact to be grounded, and by IDG each fact is grounded, so it is not the case that some fact cannot be grounded because of IDG's regress. ${ }^{23}$

I conclude that IDG does not create a vicious regress of the appropriate kind, and hence a defense of the controversial premise (that if all facts have ground, there are no facts) based on IDG creating a vicious regress of the appropriate kind fails.

\section{Against grounding being well-founded}

Here is what I take to be the best argument for why SNG need not obey WF, i.e. why it is possible that SNG is true, but WF is false.

First, assume proper parthood is a strict partial order, and that the concrete world $U$ is gunky: every entity in $U$ has a proper part. ${ }^{24}$ Then $U$ is indefinitely divisible into proper parts, i.e. unlimitedly so (so U contains neither extended nor unextended mereological atoms). Assume further that for any $\mathrm{x}$, if $\mathrm{x}$ is part of (or in) $U$, then the fact that x exists is (at least partly) grounded in the fact that its proper parts exist. Then we have in effect a needed case of IDG, contradicting WF (cf. Rosen, 2010:116). So, if SNG must obey WF, then either proper parthood is not a strict partial order or such a gunky scenario is

\footnotetext{
${ }^{23}$ See also Schaffer (forthcoming:section 4.5). My arguments above amounts to a rejection of Schaffer's "transfer model" in the sense that I argue there is no need for a source of being.

24 On gunk, see e.g. Sider (1993), Schaffer (2003), and Arntzenius (2008).
} 
metaphysically impossible; but proper parthood is a strict partial order and such a gunky scenario is metaphysically possible, so, SNG need not obey WF.

Second, assume proper parthood is a strict partial order, and that the concrete world $U$ is junky: every entity in $U$ is a proper part. ${ }^{25}$ Then $U$ is indefinitely extendable along the proper parthood chains, i.e. unlimitedly so (so U contains no maximal fusion that is not a proper part). Assume further that for any $x$ and $y$ in $U$, if $x$ is a proper part of $y$, then the fact that $x$ exists is (at least partly) grounded in the fact that $y$ exists. ${ }^{26}$ Then we have in effect another case of IDG, contradicting WF. So, if SNG must obey WF, then either proper parthood is not a strict partial order or such a junky scenario is metaphysically impossible; but proper parthood is a strict partial order and such a junky scenario is metaphysically possible (Bohn, 2009b), so SNG need not obey WF.

Finally, assume proper parthood is a strict partial order, and that the concrete world $U$ is hunky: every entity in $U$ both is and has a proper part. ${ }^{27}$ Then $\mathrm{U}$ is indefinitely extendable and indefinitely divisible along the proper parthood chains (so $U$ contains neither a minimal nor a maximal entity). Then, whichever mereological direction a chain of grounding goes (though of course it cannot go in both directions at once!), we have in effect another case of IDG, contradicting WF. So, if SNG must obey WF, then either proper parthood is not a strict partial order or such a hunky scenario is metaphysically impossible; but proper parthood is a strict partial order and such a hunky scenario is metaphysically possible (Bohn, 2009b, 2012), so SNG need not obey WF.

These three arguments seem very good to me; in fact, they seem as good as arguments get in philosophy. Rhetorically: why exactly is a gunky, a junky or a hunky scenario metaphysically impossible? I know of no convincing reason to believe they are. ${ }^{28,29}$

Assuming SNG appropriately tracks the mereological hierarchy as above (which is no minor assumption, but we only need its mere possibility here), I conclude that the claim that SNG must obey WF is simply false.

In fact, there are even some (highly defeasible!) reasons to believe that the concrete world is actually hunky. We are faced with a general cosmic pattern that so far has no clear end points in sight. Starting high up, the universe is partly composed of clusters of galaxies; the clusters of galaxies are partly composed of galaxies; the galaxies are partly composed of solar systems; the solar systems are partly composed of planets and stars; the planets and stars are partly composed of various chemicals; the various chemicals are partly composed of molecules; the various molecules are partly composed of atoms; the atoms are partly composed of electrons, protons and neutrons; the protons and neutrons are

\footnotetext{
25 On junk, see Schaffer (2010) and Bohn (2009a; 2009b; 2012; ms).

${ }^{26}$ See Schaffer (2010), where this direction of grounding is defended. If junk is possible, Schaffer's version of WF in terms of priority monism fails to be necessarily true (Bohn, 2012).

27 On hunk, see Bohn (2009b; 2012).

${ }^{28}$ Arguably, Sider's $(2011,2013)$ reasons rest on a too deflationary notion of modality.

${ }^{29}$ In Bohn $(2009 a, 2009 b, 2012)$ I argued that if composition is not identity and gunk is possible, then junk is possible. I now believe (i) that composition is identity and entails unrestricted composition (Bohn, 2009c, 2011, 2014); (ii) that hunk is possible, likely actual, if not necessary; (iii) that plural comprehension is false; and (iv) that because of (iii), (i) and (ii) are compatible (Bohn, ms).
} 
partly composed of various quarks; the various quarks are partly composed of ... to be continued? Starting low down, the various quarks partly compose the protons and neutrons; the electrons, protons and neutrons partly compose atoms; the atoms partly compose molecules; the molecules partly compose chemicals; the chemicals partly compose planets and stars; the planets and stars partly compose solar systems; the solar systems partly compose galaxies; the galaxies partly compose clusters of galaxies; the clusters of galaxies partly compose super-clusters of galaxies; the super-clusters of galaxies partly compose ... to be continued? Or perhaps they partly compose the universe, which in turn partly compose a multiverse; ${ }^{30}$ which in turn partly compose ... to be continued?

As science has progressed, we have again and again discovered that $U$ is both bigger (cf. the development of cosmology) and smaller (cf. the development of particle physics) than we thought before. Considering that overall cosmic pattern, we are faced with some inductive/abductive reasons to think there is no end in either direction; dismissing these reasons out of hand, and especially on a priori grounds, seems scientifically and theoretically irresponsible. ${ }^{31}$

By extrapolation on the above cosmic pattern, we are simply forced to question whether $\mathrm{U}$ is in fact open ended in both directions, and, arguably, we have some inductive/abductive reason to think it is, no convincing reason to think it's not. First, arguably, there is neither a convincing a posteriori reason nor a convincing a priori reason for believing that the cosmic pattern does eventually have a stopping point; at least none that cannot be equally well explained as being our (hopeful) theoretical idealization rather than a real existent. ${ }^{32}$ Second, concerning the junk-direction in particular, there is, again arguably, neither a convincing a posteriori reason nor a convincing a priori reason to believe there is an all-encompassing infinite whole rather than infinitely many bigger and bigger wholes. ${ }^{33}$ Third, concerning the gunk direction in particular, there is, again arguably, neither a convincing a posteriori reason nor a convincing a priori reason to believe there are mereological atoms. ${ }^{34}$ Rhetorically, why think an end point is anything but our (hopeful) theoretical idealization in any case?

So, there are some reasons to think the concrete world is actually hunky, not just merely possibly so. If so, again assuming SNG appropriately tracks the mereological hierarchy, SNG even actually fails to obey WF (no matter which direction a grounding chain goes).

Of course, all this leaves open whether there might be fundamentality somewhere else in the mereological hierarchy than at the top or at the bottom, or whether there might be fundamentality in a way that fails to appropriately

\footnotetext{
30 Cf. Carr (2009).

31 See also Schaffer (2003), which provides a similar inductive argument for gunk in particular. Note that, contra Sider $(2011,2013)$, the above inductive/abductive reasons are not just a handful of cases of unpacking particles, but rather they are based on a much more general cosmic pattern. Note also that Sider's $(2011,2013)$ other criticisms of the actuality of gunk rest on the assumptions that fundamentality is an all-or-nothing matter and that fundamentality is well-founded; both assumptions are rejected by our picture of SNG+IDG.

32 Of course, appealing to $\mathrm{WF}$ at this point is a non-starter.

33 See Bohn (2009b) and Carr (2009).

34 See e.g. Arntzenius (2008).
} 
track the mereological structures at all. Raven (forthcoming) defends one such alternative, where there can be fundamentality without a foundation a la WF. The core idea is that fundamentality amounts to ineliminability from a grounding chain, rather than being a fact at an end-point of it. As such, there can, at least in one sense, be fundamentality even if the world is hunky. Raven's account is subtle, and I cannot for reasons of space adequately discuss it here, but merely note two things: first, the fundamental is then not necessarily facts, so we are beyond SNG, and second, it seems even such a watered-down notion of fundamentality can be indefinitely descending, leaving us with nothing truly fundamental at all. 35

Life is short, with no time to take deep breaths, so let's move on to some other arguments. As we saw earlier, WF and IDG are mutually incompatible, so the proponent of WF must deny IDG (and proponents of IDG must deny WF). Given that grounding is explanatory (as per SNG), IDG is equivalent to a statement of what we might call the metaphysical principle of sufficient reason (MPSR): every fact $p$ has a metaphysical explanation (see Guigon, 2014; cf. Della Rocca, 2010). So, the proponent of WF must deny MPSR too. That should not be too surprising: WF postulates that there are some facts that have no ground, so by SNG neither do they have a metaphysical explanation.

But consider what we might call the well-founded grounding riddle: assume WF, i.e. that all grounding chains end in some fundamental, ungrounded facts. Consider these ungrounded facts. Either they have a metaphysical explanation (as per SNG) or they don't. If they do, they are of course not ungrounded, in which case grounding is not well-founded after all. If they don't, then they have no ground. But then the obvious question arises: whence these fundamental facts? ${ }^{36}$

Not being able to answer this question fails to provide a natural resting point for thought. ${ }^{37}$ There seems to be at least three kinds of answers. First, Brutalism: the ungrounded facts just don't have an explanation, and there is no explanation for it beyond that. Second, Indefinitism: there are no ungrounded facts because every fact has an indefinitely descending ground in all directions, so the riddle never arises. Third, Loopism: there are no ungrounded facts because if you go far enough down any grounding chain you'll end up where you started. ${ }^{38}$

But just like not answering the above question is no natural resting point for thought, so Brutalism too is no natural resting point for thought. This is so because there is no non-ad hoc way to draw the line between facts that do and do not have a metaphysical explanation, and drawing an ad hoc line is surely no

\footnotetext{
35 Raven (forthcoming) does not deny this second point.

36 Note that this latter question is what I earlier called a local question (LQ), not a regional (RQ) or a global question (GQ). It resembles the most common objection to Aquinas' foundationalism: if God is the cause of all things, what caused God?

37 Thanks to Ralph Henk Vaags.

38 For a defense of Brutalism, see e.g. Aquinas, T. (1266-8/1993); Schaffer (2009; 2010; forthcoming); for a defense of Loopism, see e.g. Bliss (2014); and for a defense of Indefinitism, see e.g. Bliss (2013); Tahko, (2014); Morganti (forthcoming); not to mention the paper you are currently reading. Note that where I use 'indefinitism', others use, misleadingly in my mind, 'infinitism'.
} 
natural resting point for thought (cf. Della Rocca, 2010). ${ }^{39}$ So, all else being equal, unless it contradicts our evidence, we should prefer Indefinitism or Loopism over Brutalism. Assuming the transitivity of grounding, Loopism contradicts the irreflexivity of grounding, so assuming SNG, according to which grounding is both transitive and irreflexive, Loopism is out. ${ }^{40}$ So, unless it contradicts our evidence, we should prefer Indefinitism over Brutalism. As argued throughout the present paper, all else is equal and Indefinitism doesn't contradict any evidence, so we should prefer Indefinitism over Brutalism. That is, on our picture, WF is Brutalism and IDG is Indefinitism, so we should prefer IDG over WF. We have assumed SNG, so we should prefer SNG+IDG over SNG+WF.

One might object that there are various alternative ways one might try to explain the ungrounded facts, making Brutalism less brute, and thus a more natural resting point for thought. ${ }^{41}$ For example, one might think it somehow lies in the essences of ungrounded facts that they are ungrounded (Rosen, 2010:128133; Dasgupta, forthcoming), which thus might somehow explain them, but in a different sense from grounding. But then again, I say, it might just not thus lie in their essences; we simply don't know what the ungrounded facts are, so we simply don't know what does and does not have an explanation in terms of their essences. As far as I can tell, we simply have no convincing reason to believe there actually is such an alternative explanation of ungrounded facts in terms of their essences.

Dasgupta (forthcoming) is perhaps the best attempt at this sort of account, according to which both MPSR and WF are true, but the fundamental facts are not apt for metaphysical explanation. But the fact that all and only the fundamental truths just happen to necessarily not be apt for metaphysical explanation is incredible. ${ }^{42}$ The idea that ungrounded facts are by their essence ungrounded is an interesting idea, but I just see no convincing reason to believe it is actually true, not to mention to rest the necessity of SNG+WF on it. The same goes, I say, in one admittedly too big sweep, for other ways of trying to alternatively explain the ungrounded (e.g. causally, or teleologically). So, it still seems to me we should prefer SNG+IDG over SNG+WF, if not only for theoretical purposes.

Note that MPSR is often thought to entail necessitism, the view according to which all actual facts are metaphysically necessary (see Della Rocca, 2010; Dasgupta, forthcoming). But, as shown in Guigon (2014), neccessitism is in fact no implication of MPSR as such, so necessitism is neither here nor there for SNG+IDG as such.

Here is a related methodological argument for SNG+IDG over SNG+WF: all else being equal, a theory that respects MPSR is better than a theory that violates MPSR; there is no particular reason to think that WF is true; there is no particular reason to think that IDG is false; and IDG respects MPSR, but WF

\footnotetext{
${ }^{39}$ On this point, among others, it is worth comparing Brutalism to Epistemic Foundationalism, but, unfortunately, I have no space to do so here. Thanks to Jonathan Schaffer and an anonymous referee for this volume for raising this question.

40 I don't want to rule out Loopism as such, but only for present purposes, where we are assuming SNG. Maybe the mental is grounded in the physical, but the physical in turn is grounded in the mental. I find such a picture of Loopism well worth exploring. 41 Thanks to Jon Litland, Alex Skiles and Kelly Trogdon for pushing me on this.

42 Thanks to Mike Raven.
} 
violates MPSR; so the theory of SNG+IDG is an overall better theory than the theory of SNG+WF. Therefore, to the extent we should prefer the better of two theories, we should prefer SNG+IDG over SNG+WF. ${ }^{43}$

The first premise is not claiming that MPSR is true, just that it is better to not violate it unless one has a good reason to. In short, neutrality and non-ad hocness are theoretical virtues. The second and third premise is basically what this whole section 2 of the paper has been defending. The fourth premise is as we have seen provable: IDG is logically equivalent with MPSR, and WF is logically incompatible with IDG, so WF is also incompatible with MPSR. ${ }^{44}$

Most generally, the proponent of WF faces the problem of having to explain a non-ad hoc restriction on MPSR, which is no easy task (cf. Della Rocca, 2010). The proponent of IDG faces no such problem; and, at least for theoretical purposes, to the extent SNG doesn't need to restrict MPSR, it shouldn't; it doesn't need to, so it shouldn't. The latter is just good old scientific and theoretical practice.

Now, contra my methodological arguments above, Cameron (2008) argues that SNG+WF is theoretically better than SNG+IDG because SNG+WF provides a more unified theory than SNG+IDG: by WF there are some fundamental facts in terms of which all others are metaphysically explained, but by IEG there is not; and a more unified theory is better than a less unified theory. But, why believe that there is more theoretical unity with fundamental facts than without? First, by going far enough down the grounding chains, there could be as much unity without fundamental facts as with. Second, the fundamental facts might come in separate pluralities having little or nothing in common, in which case there could be as much disunity with fundamental facts as without. That is, there is a big difference between saying that some fundamental facts ground all else and saying that all non-fundamental facts are grounded in some fundamental facts. The former might bring about more unity, but the latter need not. I have construed things in terms of the latter, not the former. Why think the former is the case? As far as I can tell, $\mathrm{SNG}+\mathrm{WF}$ thus need not provide any more theoretical unity than SNG+IDG. ${ }^{45}$

\section{Bibliography}

- $\quad$ Aquinas, T. (1266-8/1993). Selected Philosophical Writings. Oxford University Press.

- Arntzenius, F. (2008). Gunk, Topology and Measure. Oxford Studies in Metaphysics, vol.4. Oxford University Press.

- Bliss, R. (2013). Viciousness and the structure of reality. Philosophical Studies 166, pp.399-418.

- $\quad$------- (2014). Viciousness and circles of ground. Metaphilosophy, Vol.45, No.2, pp. 245-256.

\footnotetext{
${ }^{43}$ See also Della Rocca (2010). Note, importantly, that we're talking about metaphysical theories here.

${ }^{44}$ Note, importantly, that I have not said that IDG is one and the same claim as MPSR.

45 Thanks to Jon Litland, Matteo Morganti, Mike Raven, Jonathan Schaffer, Kelly Trogdon, Alex Skiles, and two anonymous referees for this volume.
} 
- $\quad$ Bliss, R. \& Trogdon, K. (2014). Metaphysical Grounding. Stanford Encyclopedia of Philosophy, Nov 25, 2014.

- Bohn, E.D. (2009a). An argument against the necessity of unrestricted composition, Analysis Vol.69, No.1, pp.25-29.

- $\quad$----------- (2009b). Must there be a top level? Philosophical Quarterly Vol.59, No.235, pp. 193-201.

- $\quad$-------- (2009c). Composition as Identity. PhD Dissertation, UMass Amherst, 2009.

- --------- (2011). Commentary on Parts of Classes. Humana.Mente 19, pp.151-158.

- $\quad$------ (2012). Monism, Emergence, and Plural Logic, Erkenntnis Vol.76, No.2, pp. 211-223.

- $\quad$------- (2014). Unrestricted Composition as Identity. In D. Baxter \& A. Cotnoir (eds.) Composition as Identity, Oxford University Press, 2014.

- $\quad$------ (ms). Plural Comprehension, Composition, Sets, and Junk.

- Cameron, R. (2008). Turtles all the way down. Philosophical Quarterly Vol.58, No.230, pp.1-14.

- Carr, B. (ed.) (2009). Universe or Multiverse? Cambridge University Press.

- Dasgupta, S. (2014a). On the Plurality of Grounds. Philosophers' Imprint Vol.14, No.20.

111.9 , pp. 557-592.

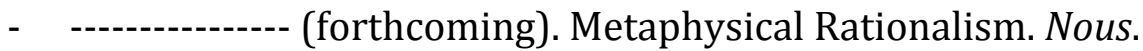

- Della Rocca, M. (2010). PSR. Philosopher's imprint, Vol. 10, No. 7.

- Dixon, T.S. (forthcoming). What is the Well-Foundedness of Grounding? Mind.

- Fine, K. (2001). The Question of Realism. Philosophers' Imprint Vol.1, No.1.

- ------ (2012). A Guide to Ground. In F. Correia \& B. Schnieder (eds.) Metaphysical Grounding: understanding the structure of reality, Cambridge University Press, 2012.

- Guigon, G. (2015). A universe of explanations. Oxford Studies in Metaphysics, vol.9. Oxford University Press.

- Hume, D. (1779/1993). Dialogues and Natural History of Religion. Oxford University Press.

- Koslicki. K. (2015). The coarse-grainedness of grounding. Oxford Studies in Metaphysics, Volume 9, Oxford University Press.

- Litland, J. (forthcoming). Grounding Ground. Oxford Studies in Metaphysics. Oxford University Press.

- Morganti, M. (Forthcoming). Dependence, Justification and explanation: Must Reality be Well-Founded? Erkenntnis.

- Nolan, D. (2001). What's wrong with infinite regresses? Metaphilosophy 32.5, pp. 523-538.

- Pruss, A.R. (2012). The Leibnizian Cosmological Argument. In W.L. Craig \& J.P. Moreland (eds.) Natural Theoology. Wiley-Blackwell, 2012.

- Rabin, G.O. \& B. Rabern (forthcoming). Well Founding Grounding Grounding. Journal of Philosophical Logic.

- $\quad$ Raven, M. (2015). Ground. Philosophy Compass 10/15, pp. 322-333.

- --------- (forthcoming). Fundamentality without Foundations. Philosophy and Phenomenological Research. 
- Rosen, G. (2010). Metaphysical Dependence: Grounding and Reduction. In B. Hale \& A. Hoffmann (eds.) (2010). Modality. Oxford University Press.

- Schaffer, J. (2003). Is there a fundamental level? Nous, 37:3, pp. 498-517.

- - ------ (2009). On What Grounds What. In Chalmers et al. (eds.) Metametaphysics. Oxford University Press, 2009.

- -------- (2010). Monism: the priority of the whole. Philosophical Review ----------- (forthcoming). Grounding in the image of causation, Philosophical Studies

- Sider, T. (1993). Van Inwagen and the possibility of gunk. Analysis 53, pp.285-289.

- - ------(2011). Writing the Book of the World. Oxford University Press.

- -------- (2013). Against Parthood. Oxford Studies in Metaphysics, vol.8.

- Tahko, T.S. (2014). Boring Infinite Descent. Metaphilosophy, Vol. 45, No. 2, pp. 257-269.

- Trogdon, K. (ms). Inheritance arguments for fundamentality. This volume.

- Wilson, J. (2014). No Work for a Theory of Grounding. Inquiry, 57: 5-6, pp. 535-579. 